\title{
A Study on Correlation of Antimicrobial Resistance Pattern and Biofilm Formation among Coagulase Negative Staphylococcus Isolates
}

\author{
Radhika Katragadda* and Sowmya A. Venkateswaran \\ Department of Microbiology, Government Medical College, Omandurar Govt. Estate, \\ Chennai, Tamilnadu, India \\ *Corresponding author
}

\begin{abstract}
A B S T R A C T
Coagulase negative Staphylococcus (CONS), once considered as a commensal has now

Keywords

Coagulase negative Staphylococcus,

Methicillin resistance,

Biofilm producers,

Antimicrobial resistance

Article Info

Accepted:

10 October 2018

Available Online:

10 November 2018

evolved as a major infectious pathogen with biofilm forming ability. This study was conducted to identify the antibiotic resistance pattern of varied CONS species in association with its biofilm forming ability. CONS isolated from various clinical samples were speciated. Their antimicrobial resistant pattern and biofilm producing ability were studied and analyzed. $7.98 \%$ of our clinical isolates were CONS, with $21.52 \%$ of them being Methicillin resistant (MR-CONS) and 78.48\% were Methicillin sensitive (MSCONS). Of the MR-CONS, S. epidermidis comprises about $70.59 \%$, followed by $S$. hemolyticus (15.69\%). $23.63 \%$ of CONS are biofilm producers, of which $60.71 \%$ are MRCONS isolates and $39.29 \%$ were MS -CONS. $73.21 \%$ of biofilm producers were $S$. epidermidis. Biofilm producers were resistant to multiple antibiotics when compared to non-biofilm producers. All the CONS isolates were sensitive to vancomycin and Linezolid. With increase in incidence of MR-CONS and biofilm formation, routine identification with their antimicrobial resistance pattern should be documented and practiced to prevent the emergence of these common commensals as super bugs.
\end{abstract}

\section{Introduction}

Coagulase Negative Staphylococcus (CONS) were once considered as commensals and their pathogenic potentials have been under debate for a long time. However CONS has been accepted as a potential pathogen, being isolated in various clinical samples from 1980 onwards (Karsten Becker et al., 2014). Today CONS represent one of the major pathogens among immunocompromised and hospitalized individuals, with a considerable impact on morbidity and mortality. Reports on surveillance data taken from the National Nosocomial Infections Surveillance System have indicated CONS as one of the five most commonly reported pathogens in hospitals (Christof VonEiff et al., 2008). Common associated risk factors include patients with prosthetic devices, foreign body intravascular catheters, post-operative wounds and immunocompromised status (Sheikh et al., 2012).

CONS represent a heterogeneous group of microbes within Genus Staphylococcus, 
identified primarily by negative coagulase test. The most common CONS isolated from hospitalized patients were S. epidermidis. $S$. saprophyticus has been identified as a known causative agent of urinary tract infection in sexually active women. Other CONS, which are now increasingly identified as causative agents of urinary tract infections, wound infections and bacteremia are S. lugdunensis, S. schleiferi, S. haemolyticus, S. warneri, S. hominis and $S$. simulans. One of the major virulence factors for CONS is their ability to adhere to polymer surfaces and induce biofilm formation. Biofilm forming CONS infections pose a major challenge to clinical microbiologists and treating physicians, as they have decreased antimicrobial susceptibility within the biofilm and also found to be more resistant to commonly used antimicrobial agents when compared to nonbiofilm producers (Rupp et al., 2010). Recently Australian scientists have isolated a strain of $S$. epidermidis from hospital environment which was found to be resistant to almost all known antibiotics, probably emerging as superbug, indicating the importance of these commensals getting converted into potential nosocomial pathogens (Anna Lavdaras, 2018).

Routine laboratory identification of CONS infection, their biofilm forming ability and their antimicrobial susceptibility pattern is very important to understand the actual impact of these potential pathogens, especially in this era of advanced medical sciences with increase in susceptible population. This study is conducted in our hospital to identify the various CONS species isolated among clinical samples, their biofilm forming ability and their antimicrobial resistance pattern.

\section{Materials and Methods}

The study was conducted over a period of six months after getting Institutional Ethical
Committee approval. Various consecutive samples like pus, wound swabs, ear swabs, throat swabs, urine, body fluids, sputum and blood received in the Microbiology laboratory for culture and sensitivity were processed according to Standard Operating procedures. Samples were inoculated onto various culture media like Nutrient agar, MacConkey agar and Blood agar by sterile technique. The plates were incubated at $37^{\circ} \mathrm{C}$ overnight and examined for any growth. The colonies of Staphylococci were identified by Gram stain and Catalase test. Those Staphylococci which gave negative result in coagulase test were identified as Coagulase Negative Staphylococci (CONS). Only pure growth of CONS isolates and also repeated isolates were included in the study.

Speciation of CONS was further done using various series of biochemical tests like Nitrate reduction test, Voges Proskauer test, Urease test, Ornithine decarboxylase test, Sugar fermentation test for Trehalose, Mannitol, Mannose, Xylose, Maltose and sucrose sugars (Winn W.C. et al., 2006). Susceptibility testing to Novobiocin and Polymyxin B was done as a routine to identify $S$. saprophyticus and $S$. epidermidis respectively, as per standard guidelines.

Antimicrobial susceptibility testing was done by Kirby Bauer disc diffusion technique, with the following antibiotics as indicated by CLSI guidelines. Ampicillin $(25 \mu \mathrm{g})$, Amoxicillin + clavulanate $(30 \mu \mathrm{g})$, Ceftriaxone $(30 \mu \mathrm{g})$, Ciprofloxacin $(5 \mu \mathrm{g})$, Erythromycin $(15 \mu \mathrm{g})$, Cotrimoxazole, Gentamicn $(5 \mu \mathrm{g})$, Amikacin $(30 \mu \mathrm{g})$ and Linezolid $(30 \mu \mathrm{g})$. Methicillin resistance was identified using cefoxitin disc $(30 \mu \mathrm{g})$. Those isolates which gave inhibitory zone of $\leq 24 \mathrm{~mm}$ were considered as sensitive and hence Methicillin sensitive CONS (MSCONS) and those which gave inhibitory zone size of $\geq 30 \mathrm{~mm}$ were considered resistant, hence Methicillin resistant CONS (MR- 
CONS). Vancomycin susceptibility was detected by agar dilution method using Brain heart infusion agar containing $4 \mu \mathrm{g} / \mathrm{ml}$ of vancomycin drug. Isolates which grow in this screen agar were considered as vancomycin resistant strains and those which fail to grow in this screen agar after overnight incubation at $37^{\circ} \mathrm{C}$ were considered as vancomycin sensitive strains (Xiao Xue Ma et al., 2011).

Biofilm formation among various clinical CONS isolates was detected by tube method. A loop full of overnight culture of CONS isolates on nutrient agar was inoculated into $10 \mathrm{ml}$ of Trypticase soy broth with $1 \%$ of glucose. These inoculated test tubes were incubated overnight at $37^{\circ} \mathrm{C}$. These culture test tubes are then decanted and washed with phosphate buffer saline. The tubes are then dried and stained with $0.1 \%$ crystal violet. Excess stain was then washed with deionized water. The tubes are then dried by keeping it in inverted position and observed for biofilm formation. Biofilm formation was considered positive when a visible film lined the wall and bottom of the tube. Ring formation at the liquid interface was not considered as biofilm formation (Nabajit Deka, 2014).

\section{Results and Discussion}

A total of 6, 427 samples were processed for culture during the six months study period, of which 2, 970 samples were culture positive (46.21\%). Of the 2, 970 clinical isolates, 237 isolates were identified as Coagulase Negative Staphylococcus (CONS) (7.98\%).

Majority of CONS samples were isolated from pus samples (41.35\%), followed by wound swab (31.22\%). CONS were also isolated from Urine $(20.68 \%)$, ear swabs $(5.06 \%)$ and sputum (1.69\%) samples. S. epidermidis was the most common species of CONS isolated from clinical samples (43.46\%), followed by S. hemolyticus (18.57\%). S. saprophyticus comprises about $69.39 \%$ of CONS isolates from urine samples.

Of all the CONS isolates $21.52 \%$ were Methicillin resistant strains (MR-CONS) and $78.48 \%$ were Methicillin sensitive strains (MS-CONS). Of the MR-CONS, S.epidermidis comprises about $70.59 \%$, followed by S.hemolyticus (15.69\%). Of the 51 MR-CONS isolates $37.25 \%$ were from pus samples, followed by $33.33 \%$ in urine samples. About $25.50 \%$ of MR -CONS were isolated from Wound swab and 3.92\% from sputum samples

Among the various 237 CONS isolates, 23.63 $\%$ of them are biofilm producers, of which $60.71 \%$ are MR-CONS isolates and $39.29 \%$ were MS -CONS. 181(76.37\%) of the isolates were non biofilm producers. Among the biofilm producers, S.epidermidis are the commonest isolate producing biofilm (73.21\%) followed by S.hemolyticus (16.07\%). About $33.3 \%$ of Methicillin resistant strains are non-biofilm producers, when compared to Methicillin sensitive strains where $88.17 \%$ of strains are non-biofilm producers

All the CONS isolates were $100 \%$ sensitive to Vancomycin and Linezoliod. Maximum antibiotic resistance was found with Ampicillin drug followed by Cotrimoxazole and Gentamicin. The isolates were found to be highly sensitive to Amikacin and Amoxyclav.

MR-CONS isolates were found to be more resistant to multiple antibiotics than MSCONS isolates. Irrevalant of Methicillin resistant or sensitive status all CONS isolates were found to be $100 \%$ sensitive to Vancomycin and Linezolid.

Biofilm producing CONS isolates showed more resistance towards commonly used antibiotics than non-biofilm producers. In 
Both biofilm and non-biofilm producer group highest resistance was seen with Ampicillin (73.21\%, 63.12\%), followed by Cotrimoxazole $(57.14 \%, \quad 52.49 \%)$ and Gentamicin (59.36\%, 54.49\%), respectively. All the CONS isolates were $100 \%$ sensitive to Vancomycin and Linezolid.

With continuing increase in the susceptible risk population, it has been acknowledged by various studies that CONS should not be considered as an accidental pathogen any more.

Many of the studies describe the clinical spectrum of CONS infection as a whole, but various species of CONS had acquired virulence factors to produce specific and severe infections. The real impact of less frequently reported CONS species may be underreported as they are not routinely speciated and reported. This study was conducted in our tertiary care hospital to find out the different species of CONS isolated from various clinical samples and to study their antibiotic resistance pattern with reference to biofilm formation.
Among the 2, 970 isolates, $7.98 \%$ (237) were identified to be CONS isolates, which is comparatively less than other studies conducted in various parts of India, where the prevalence rates of CONS isolates vary from $14.3 \%$ to $44.8 \%$ (Lubna samad et al., 2017) (Khadri et al., 2010) (Al Mazroea, 2009) (Mir et al., 2013) (Table 1). Majority of CONS in our study were isolated from pus samples (41.35\%), followed by wound swab (31.22\%). $20.68 \%$ of urine isolates, $5.06 \%$ of ear swab isolates and $1.69 \%$ of sputum sample isolates were found to CONS. Studies conducted by Lubna Samad et al., (2017) and Roopa et al., (2015) also reveals that CONS were more commonly isolated from pus samples followed by wound swabs and urine samples. They have also documented CONS isolates from catheter tips, blood and body fluids, indicating the varied spectrum of clinical infection that can be caused by CONS. Study conducted by Nahed A. Al Laham et al., (2017) had also documented that the most common clinical source of CONS in their study was pus (34.6\%), followed by wound swabs (23.5\%), urine $(18.5 \%)$ and blood (14.8\%) (Table 1).

Table.1 Common CONS species isolated from various clinical samples

\begin{tabular}{|c|c|c|c|c|c|c|c|c|c|c|c|c|c|}
\hline \multirow[t]{2}{*}{$\begin{array}{l}\text { S. } \\
\text { No. }\end{array}$} & \multirow[t]{2}{*}{$\begin{array}{l}\text { CONS species / } \\
\text { Clinical sample }\end{array}$} & \multicolumn{2}{|c|}{$\begin{array}{c}\text { Pus } \\
(\mathrm{n}=98)\end{array}$} & \multicolumn{2}{|c|}{$\begin{array}{l}\text { Wound } \\
\text { swabs } \\
(\mathrm{n}=74)\end{array}$} & \multicolumn{2}{|c|}{$\begin{array}{l}\text { Urine } \\
(n=49)\end{array}$} & \multicolumn{2}{|c|}{$\begin{array}{c}\text { Ear swabs } \\
(\mathbf{n}=12)\end{array}$} & \multicolumn{2}{|c|}{$\begin{array}{c}\text { Sputum } \\
(\mathrm{n}=4)\end{array}$} & \multicolumn{2}{|c|}{$\begin{array}{c}\text { Total } \\
(\mathbf{n}=\mathbf{2 3 7})\end{array}$} \\
\hline & & No & $\%$ & No & $\%$ & No & $\%$ & No & $\%$ & No & $\%$ & No & $\%$ \\
\hline 1 & S.epidermidis & 46 & 46.94 & 38 & 51.35 & 10 & 20.41 & 7 & 58.33 & 2 & 50 & 103 & 43.46 \\
\hline 2 & S.hemolyticus & 21 & 21.43 & 17 & 22.97 & 3 & 6.12 & 2 & 16.67 & 1 & 25 & 44 & 18.57 \\
\hline 3 & S.hominis & 14 & 14.28 & 11 & 14.86 & - & - & - & - & - & - & 25 & 10.55 \\
\hline 4 & S.saprophyticus & - & - & - & - & 34 & 69.39 & - & - & - & - & 34 & 14.34 \\
\hline 5 & S.schleiferi & 10 & 10.20 & 7 & 9.46 & - & - & 3 & 25 & 1 & 25 & 21 & 8.86 \\
\hline 6 & S.warneri & 7 & 7.14 & 1 & 1.35 & 2 & 4.08 & - & - & - & - & 10 & 4.22 \\
\hline
\end{tabular}


Table.2 Distribution of MR -CONS and MS-CONS among the CONS isolates

\begin{tabular}{|c|c|c|c|c|c|}
\hline \multirow[t]{2}{*}{ CONS isolates } & \multicolumn{2}{|c|}{ MR- CONS $(n=51)$} & \multicolumn{2}{|c|}{ MS- CONS $(n=186)$} & \multirow[t]{2}{*}{ Total } \\
\hline & No & $\%$ & No & $\%$ & \\
\hline S. epidermidis & 36 & 70.59 & 67 & 36.02 & 103 \\
\hline S. hemolyticus & 8 & 15.69 & 36 & 19.35 & 44 \\
\hline S. hominis & 3 & 5.88 & 22 & 11.83 & 25 \\
\hline S. saprophyticus & 1 & 1.96 & 33 & 17.74 & 34 \\
\hline S. schleiferi & 2 & 3.92 & 19 & 10.22 & 21 \\
\hline S. warneri & 1 & 1.96 & 9 & 4.84 & 10 \\
\hline Total & 51 & 100 & 186 & 100 & 237 \\
\hline
\end{tabular}

Table.3 Distribution of biofilm producers among the CONS isolates

\begin{tabular}{|l|c|c|c|c|c|}
\hline & \multicolumn{2}{|c|}{ CONS isolates } & \multicolumn{2}{|c|}{$\begin{array}{c}\text { Methicillin sensitive } \\
\text { (186) }\end{array}$} & Total \\
\cline { 2 - 6 } & $\begin{array}{c}\text { Biofilm } \\
\text { producers } \\
(\mathbf{3 4 )}\end{array}$ & $\begin{array}{c}\text { Non biofilm } \\
\text { producers } \\
(\mathbf{1 7 )}\end{array}$ & $\begin{array}{c}\text { Biofilm } \\
\text { producers } \\
\mathbf{( 2 2 )}\end{array}$ & $\begin{array}{c}\text { Non biofilm } \\
\text { producers } \\
(\mathbf{1 6 4 )}\end{array}$ \\
\hline S. epidermidis & 24 & 12 & 17 & 50 & 103 \\
\hline S. hemolyticus & 6 & 2 & 3 & 33 & 44 \\
\hline S. hominis & 1 & 2 & - & 22 & 25 \\
\hline S. saprophyticus & 1 & - & 2 & 31 & 34 \\
\hline S. schleiferi & 1 & 1 & - & 19 & 21 \\
\hline S. warneri & 1 & - & - & 9 & 10 \\
\hline Total & 34 & 17 & 22 & 164 & 237 \\
\hline
\end{tabular}

Table.4 Antibiotic resistance pattern of CONS isolates

\begin{tabular}{|c|c|c|c|c|c|c|c|c|c|c|c|c|}
\hline \multirow[t]{2}{*}{$\begin{array}{l}\text { Antibiotics/ } \\
\text { Cons isolates }\end{array}$} & \multicolumn{2}{|c|}{$\begin{array}{l}\text { S.epidermidis } \\
(\mathrm{n}=103)\end{array}$} & \multicolumn{2}{|c|}{$\begin{array}{l}\text { S.hemolyticus } \\
(n=44)\end{array}$} & \multicolumn{2}{|c|}{$\begin{array}{l}\text { S.hominis } \\
(n=25)\end{array}$} & \multicolumn{2}{|c|}{$\begin{array}{l}\text { S.saprophyticus } \\
(\mathrm{n}=34)\end{array}$} & \multicolumn{2}{|c|}{$\begin{array}{l}\text { S.schleiferi } \\
(n=21)\end{array}$} & \multicolumn{2}{|c|}{$\begin{array}{l}\text { S.warneri } \\
(\mathrm{n}=10)\end{array}$} \\
\hline & No & $\%$ & No & $\%$ & No & $\%$ & No & $\%$ & No & $\%$ & No & $\%$ \\
\hline Ampicillin & 78 & 75.73 & 29 & 65.90 & 16 & 64 & 20 & 58.82 & 11 & 52.38 & 5 & 50 \\
\hline Amoxyclav & 23 & 22.33 & 11 & 25 & 4 & 16 & 4 & 11.76 & 3 & 14.28 & 1 & 10 \\
\hline Ceftriaxone & 30 & 29.12 & 13 & 29.54 & 5 & 20 & 8 & 23.53 & 5 & 23.81 & 2 & 20 \\
\hline Ciprofloxacin & 39 & 37.87 & 15 & 34.09 & 6 & 24 & 8 & 23.53 & 6 & 28.57 & 2 & 20 \\
\hline Erythromycin & 54 & 52.42 & 22 & 50 & 12 & 48 & 15 & 44.12 & 18 & 38.10 & 3 & 30 \\
\hline Cotrimoxazole & 62 & 60.19 & 26 & 59.09 & 13 & 52 & 18 & 52.94 & 10 & 47.62 & 5 & 50 \\
\hline Gentamicin & 63 & 61.16 & 27 & 61.36 & 14 & 56 & 19 & 55.88 & 11 & 52.38 & 5 & 50 \\
\hline Amikacin & 27 & 26.21 & 11 & 25 & 6 & 24 & 2 & 5.88 & 2 & 9.52 & 0 & 0 \\
\hline
\end{tabular}


Table.5 Antibiotic resistance pattern of MR -CONS and MS-CONS

\begin{tabular}{|l|c|c|c|c|}
\hline \multirow{2}{*}{ CONS isolates } & \multicolumn{2}{|c|}{ MR-CONS $(\mathbf{n = 5 1 )}$} & \multicolumn{2}{c|}{ MS-CONS (n=186) } \\
\hline & No & \% & No & \% \\
\hline Ampicillin & 38 & 74.51 & 116 & 62.37 \\
\hline Amoxyclav & 10 & 19.61 & 26 & 13.98 \\
\hline Ceftriaxone & 15 & 29.41 & 45 & 24.19 \\
\hline Ciprofloxacin & 18 & 35.29 & 52 & 27.96 \\
\hline Erythromicin & 26 & 50.98 & 74 & 39.78 \\
\hline Cotrimoxazole & 30 & 58.82 & 98 & 52.69 \\
\hline Gentamicin & 31 & 60.78 & 104 & 55.91 \\
\hline Amikacin & 12 & 23.52 & 18 & 9.67 \\
\hline
\end{tabular}

Chart.1 Antibiotic resistance pattern of CONS isolates, in correlation with biofilm production

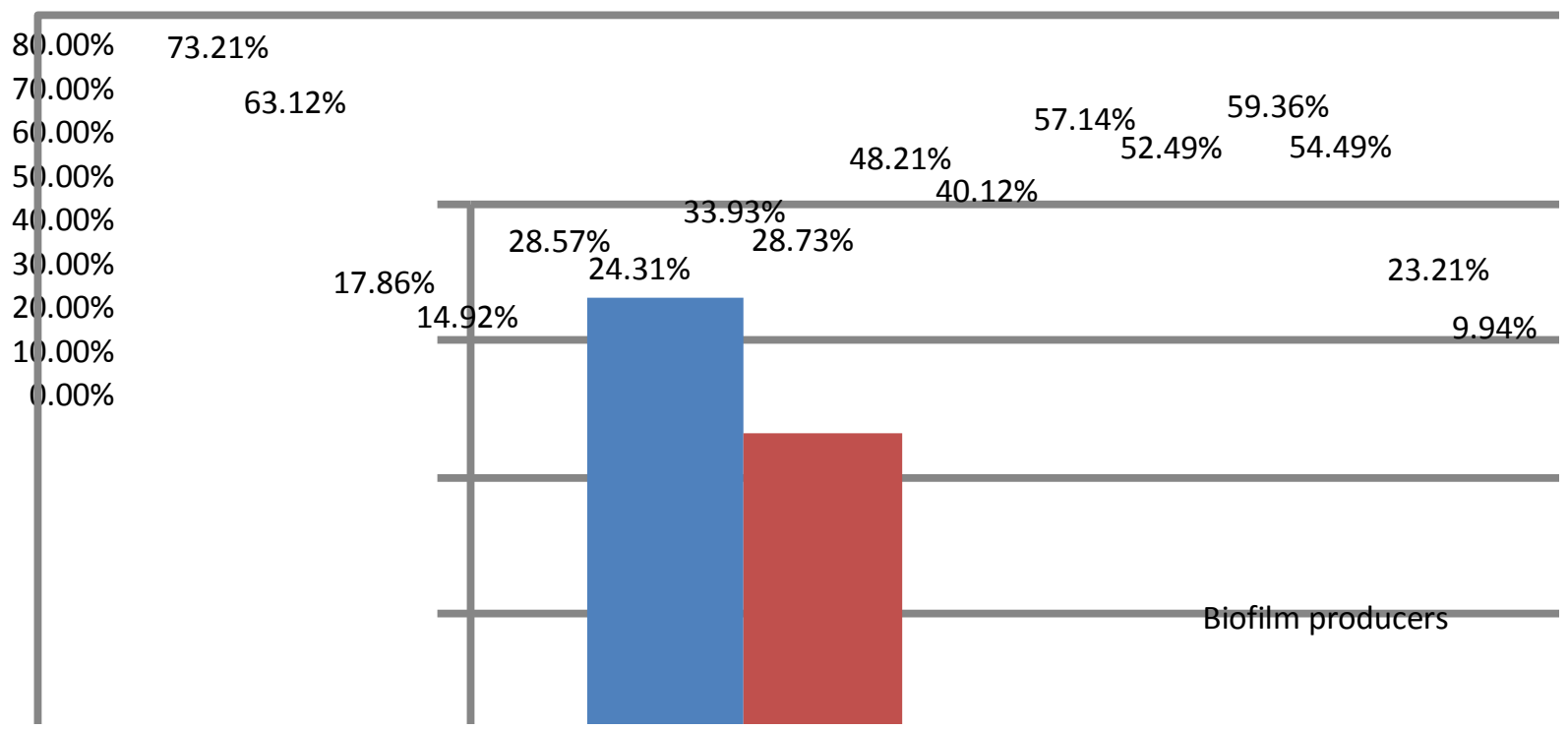

The most common species of CONS that was isolated from various clinical samples, in our study were $S$. epidermidis (43.46 \%), followed by $S$. hemolyticus (18.57\%). Other clinically significant CONS isolates were $S$. hominis $(10.55 \%)$, S. schleiferi $(8.86 \%)$ and S. warneri (4.22\%). The most common species isolated from urine samples was $S$. saprophyticus $(14.34 \%)$. Study conducted by Roopa et al., (2015) also reveals the same pattern of CONS species distribution as in our study with $50.8 \%$ of isolates being S.epidermidis, $26.7 \%$ S.hemolyticus, $7.1 \%$ of S.schleiferi and $4.46 \%$ of S.saprophyticus.
However study conducted by Sheikh A.F. et al., (2012) have documented 16 species of CONS isolates, commonest being S.epidermidis (29.4\%) and S.hemolyticus (14.9\%), along with uncommon species like S.arlettae, S.auricularis and S.caprae species. This indicates the wide varied spectrum of CONS isolates that can cause significant clinical infection (Table 1).

In urine samples the commonest CONS species isolated was S.saprophyticus $69.39 \%$ followed by S.epidermidis $20.41 \%$. S. saprophyticus has been considered as a true 
urinary pathogen for a long period of time as it may be related to carriage of the organism in the rectum or introitus. Winn et al., (2006) had revealed S.saprophyticus as the second commonest cause of urinary tract infections in females, next to E.coli (Table 2).

About $21.52 \%$ of CONS isolates in our study were MR-CONS, which is less compared to study conducted by Puneet Bhatt et al., (2016) and Bilal Ahmad Mir et al., (2013) who had documented $32.7 \%$ and $40 \%$ MR-CONS isolates from various clinical samples respectively. Nearly $70.59 \%$ of MR-CONS isolates in our study was S.epidermidis, followed by S.hemolyticus (15.69\%). Other CONS species contribute to about $5.88 \%$ $1.96 \%$ of MR-CONS isolates. Similar results are shown in a South Indian study (Saravanan Murugesan et al., 2015) where $40 \%$ of S.epidermidis, $28 \%$ of S.hemolyticus, $20 \%$ of S.hominis and $12 \%$ of S.warneri were methicillin resistant. However results obtained by Rathanin Seng et al., (2017) had documented $41.1 \%$ of S.hemolyticus, $30.1 \%$ of S.epidermidis and $28.8 \%$ of other CONS species as methicillin resistant

Of the 51 MR-CONS isolates $37.25 \%$ were from pus samples, followed by $33.33 \%$ in urine samples. About $25.50 \%$ of MR -CONS were isolated from Wound swab and $3.92 \%$ from sputum samples. Study conducted by C. Roopa et al., (2015) and Chincholkar et al., (2017) also reveals that majority of drug resistance CONS were isolated from pus samples. As S.epidermidis was the major pathogen isolated from pus samples and as majority of MR-CONS was isolated from pus samples, S.epidermidis has become one of the significant CONS species exhibiting methicillin resistance and may be emerging as an important multidrug resistant pathogen of concern. Various scientific studies worldwide reveal nearly $80-90 \%$ of S.epidermidis are Methicillin resistant carrying mec A gene.
Study conducted by Francois Barbier et al., (2010) among various CONS isolates exhibiting mec $A$ gene, had documented that $69.95 \%$ of S.epidermidis, $13.3 \%$ of S.homins and $12 \%$ of S.hemolyticus were methicillin resistant and it correlates with the results of our study also. Whereas study conducted by Nigeria Ezekiel Akinkunmi et al., (2010), had documented S.hemolyticus as the most common MR-CONS (46.2\%), followed by S.epidermidis $(35.6 \%)$. These differences in MR CONS isolates may be due to the difference in the species predominating the various clinical setup and variations in the species colonizing human skin and mucosa (Table 3).

Among the various CONS isolates, $23.63 \%$ are biofilm producers and $76.37 \%$ of the isolates were non biofilm producers. The biofilm producers in our study $(23.63 \%)$ were comparatively low than the result documented by Lal Bhadur shreshtha et al., (2017) and Rupp et al., (2010), who reveals that $65.38 \%$ and $80 \%$ of their CONS isolates were biofilm producers, respectively. The higher percentage of detection of biofilm producers in the above mentioned studies may be due to their utilization of additional microtitre plate method and Congo red method for biofilm detection. Majority of MR-CONS in our study were biofilm producers $(66.66 \%)$ when compared to MS-CONS (11.83\%) and this difference is statistically significant. Other studies also reveal MR-CONS as a common biofilm producer with results ranging from as high as $90.8 \%$ by R Seng et al., (2017) to $43.75 \%$ by Martini et al., (2016). Also Chincholkar et al., (2017) showed that $83.5 \%$ of biofilm producers were Methicillin resistant and Samant et al., (2012) with 60\% of MR-CONS being biofilm producers. This increased frequency of biofilm formation in MR-CONS may be due to FnbB gene mediated biofilm development among MRSA trait (Jeong ok Cha et al., 2013) (Table 3). 
Among the biofilm producers, S.epidermidis were the commonest isolate producing biofilm (73.21\%) followed by S.hemolyticus (16.07\%). Among S.saprophyticus $5.36 \%$ isolates and $1.79 \%$ isolates of S.hominis, S.schleiferi and S.warneri each were biofilm producers. Several studies conducted worldwide (Robert D. Wojtyczka et al., 2014) (Alcaraz et al., 2003) had documented S.epidermidis as a major biofilm producer with prevalence ranging from $81 \%$ to $57.7 \%$. Study conducted in India (Sardar et al., 2015) also show S.epidermidis as the predominant biofilm producer. In contrast to our study Chincholkar et al., (2017) showed S.saprophyticus as the most common biofilm producer $(52.78 \%)$ predominantly isolated from urine samples, followed by S.hemolyticus $(41.18 \%)$ and S.epidermidis (37\%). In majority of the studies the most common urine CONS isolate was S.saprophyticus and they are one of the major biofilm producers (Chincholkar et al., 2017; Alcaraz et al., 2003) (Table 4).

All the CONS species isolated in our study were $100 \%$ sensitive to Vancomycin and Linezoliod. Maximum antibiotic resistance was found with Ampicillin drug (68.44\%) followed by Gentamicin (58.35\%) and Cotrimoxazole $(55.76 \%)$. The isolates were also found to be resistant to Erythromycin (45.38\%), Ciprofloxacin (31.63\%), Ceftriaxone (26.80\%), AmoxyClav (16.80\%) and Amikacin (16.60\%).Various studies (Mir et al., 2013; Roopa et al., 2015; Lok Bahadur Shreshtha et al., 2017; Chincholkar et al., 2017) show similar resistance patterns like our study with resistance to Ampicillin ranging from $80 \%$ to $48 \%$, Gentamicin $80 \%$ to $43.8 \%$, Cotrimoxazole $75.2 \%$ to $37.5 \%$, Ceftriaxone $65 \%$ to $27.6 \%$, Erythromycin $83 \%$ to $27.6 \%$, Ciprofloxacin $60 \%$ to $12.5 \%$, Amoxyclav $21.3 \%$ to $27.6 \%$ and Amikacin $28 \%$ to $12.5 \%$. This infers that CONS species were developing resistance to multitude of antibiotics at an alarming rate, posing a difficult situation to treating clinicians (Table 5; Chart 1).

In our current study MR-CONS and biofilm producers were found to be more resistant to multiple antibiotics than MS-CONS and nonbiofilm producers. In Both biofilm and nonbiofilm producer group highest resistance was seen with Amoxicillin (73.21\%, 59.12\%), followed by Cotrimoxazole $(57.14 \%, 52.49 \%)$ and Gentamicin (59.36\%.54.49\%), respectively. All the CONS isolates were $100 \%$ sensitive to Vancomycin and Linezolid. Chincholkar et al., (2017) and Samant et al., (2012) in their study reported that biofilm producing CONS were found to be more resistant to almost all classes of antibiotics as compared to biofilm non producers. The heightened resistance to multiple antibiotics among biofilm producers may be due to the fact of slower rates of metabolism, thereby reducing their sensitivity to antibiotics. Also the presence of biofilm increases the virulence of the organism by protecting them from phagocytosis and decreasing chemotaxis in vivo, thereby inhibiting host defense mechanism. It has been documented that presence and expression of mecA gene seems to be enhanced in biofilm producers, enabling it to be resistant to multiple groups of antibiotics (Frebourg et al., 2000). However in contrast to most other studies Robert D. Wojtyczka et al., (2014) had reported that there are no significant differences in antimicrobial susceptibility between biofilm and non-biofilm producers.

Coagulase negative Staphylococci have been increasingly documented as a potential pathogen in recent years. Ability to produce biofilms and thereby emergence of drug resistance were considered as an important virulence factors. Biofilm producing CONS act as potential reservoirs leading to chronic infections. S.epidermidis has emerged as a 
most important pathogen due to its multidrug resistance and biofilm ability and soon may present itself as a superbug worldwide. Hence identification, speciation and routine monitoring of drug resistance pattern of CONS isolates becomes mandatory to prevent serious burden to the health care system caused by CONS infections.

\section{References}

Alcaraz LE, Satoress SE, Lucero RM, Puig de Centorbi ON, 2003. Species identification, slime production and oxacillin susceptibility in coagulase negative staphylococci isolated from nosocomial specimens. Braz J Microbiol. 34:45-51.

Al-Mazroea AH, 2009. Incidence and clinical significance of coagulase negative staphylococci in blood. J T U Med Sci. 4: 137-47.

Chincholkar V, Lomte A, Nilekar S, More S, 2017. Biofilm production among coagulase negative staphylococci and its correlation with antimicrobial susceptibility pattern. Med Pulse International Journal of Microbiology. Volume 3, Issue 2, Pp. 01-05.

Christof Von Eiff, Christine Heilmann, Georg Peters, 2008. Staphylococcus epidermidis: why is it so successful? Clinical Microbiology and Infection. Volume 4, Issue 6; Pp. 297-300.

Francois Barbier, Etienne Ruppe, David Hernandez, David Lebaeux, Patrice Francois, Benjamin Felix et al., 2010. Methicillin resistant Coagulase negative Staphylococci in the community: High homology of SCC mec IV a between Staphylococcus epidermidis and major clones of Methicillin resistant Staphylococcus aureus. The Journal of Infectious Disease, Volume 202, issue 2, Pages 270-281.
Frebourg NB, Lefebvre S, Baert S, Lemeland JF, 2000. PCR-based assay for discrimination between invasive and contaminating Staphylococcus epidermidis strains. Journal of Clinical Microbiology. 38(2):877-880.

Jeong-Ok Cha, Jae Il Yoo, Jung Sik Yoo, Hae-Sun Chung, Sun-Hee Park, Hwa Su Kim, Yeong Seon Lee, and Gyung Tae Chung, 2013. Investigation of Biofilm Formation and its Association with the Molecular and Clinical Characteristics of Methicillin-resistant Staphylococcus aureus. Osong Public Health Res Perspect. 4(5): 225-232.

Karsten Becker, Christine Heilman, Georg Peters, 2014.Coagulase Negative Staphylococci. Clinical Microbiology Reviews. Vol. 27; No.4; 870-926.

Khadri H, Alzohairy M, 2010. Prevalence and antibiotic susceptibility pattern of methicillin-resistant and coagulasenegative staphylococci in a tertiary care hospital in India. Int J Med Med Sci. 2: 116-20.

Lok Bahadur Shrestha, Narayan Raj Bhattarai and Basudha Khanal, 2017. Antibiotic resistance and biofilm formation among coagulase-negative staphylococci isolated from clinical samples at a tertiary care hospital of eastern Nepal. Antimicrobial Resistance \& Infection Control. 6:89

Lubna Samad, Dalip K, Kakru, Bashir A. Fomda, Shugufta Roohi, Mohd Suhail Lone. Prevalence \& risk factors associated with coagulase negative staphylococcus infections in a tertiary care centre in North India, 2017.Int J Cur Res Rev. Vol 9, issue 7, pg 39-43.

Martini R, Horner R, Rampelotto RF, Garzon LR, Nunes MS, Teixeira MD, et al., 2016. Investigation of biofilm formation in coagulase-negative staphylococci isolated from platelet concentrates 
bags. Rev Inst Med Trop Sao Paulo. 58:1.

Mir BA, Srikanth D, 2013. Prevalence and antimicrobial susceptibility of methicillin resistant Staphylococcus aureus and Coagulase negative staphylococci in a tertiary care hospital. Asian J Pharm Clin Res. 6: 231-34.

Nabajit Deka, 2014. Comparison of Tissue Culture plate method, Tube Method and Congo Red Agar Method for the detection of biofilm formation by Coagulase Negative Staphylococcus isolated from Non-clinical Isolates, Int.J.Curr.Microbiol.App.Sci.3(10) 810815.

Nahed A. Al Laham, Emad Abou Elkhair, Abdallah Bashir, Nahed Abdelateef, 2017. Resistance profiles and biofilm formation of coagulase negative staphylococci isolated from clinical specimens in a tertiary care hospital in Palestine. The International Arabic Journal of Antimicrobial agents, Vol 7, No.34.

Nigeria Ezekiel Olugbenga Akinkunmi and Adebayo Lamikanra, 2010. Species Distribution and Antibiotic Resistance in Coagulase-negative Staphylococci Colonizing the Gastrointestinal Tract of Children in Ile-Ife. Trop J Pharm Res, 9(1):39.

Puneet Bhatt, Kundan Tandel, Alina Singh, M.Kumar, Naveen Grover, A.K. Sahni, 2016. Prevalence and molecular characterization of methicillin resistance among Coagulase negative Staphylococci at a tertiary care centre. Med J Armed Forces India. 72(suppl 1) S54-S58.

Rathanin Seng, Thawatchai Kitti, Rapee Thummeepak, Phattaraporn Kongthai, Udomluk Leungtongkam, Surat Wannalerdsakun, 2017. Biofilm formation of methicillin-resistant coagulase negative staphylococci (MR-
CONS) isolated from community and hospital environments. PLOS One, 12(8): 0184172.

Robert D. Wojtyczka, Kamila Orlewska, Małgorzata Kępa, Danuta Idzik, Arkadiusz Dziedzic, Tomasz Mularz, Michał Krawczyk, Maria Miklasińska and Tomasz J. Wasik, 2014. Biofilm Formation and Antimicrobial Susceptibility of Staphylococcus epidermidis Strains from a Hospital Environment. Int. J. Environ. Res. Public Health. 11, 4619-4633.

Roopa, C., Sunilkumar Biradar, 2015. Incidence and speciation of Coagulase negative Staphylococcus isolates from clinically relevant specimens with their Antibiotic susceptibility patterns. Int.J.Curr.Microbiol.App.Sci, 4(9): 975980.

Rupp ME, Fey PD, 2010. Staphylococcus Epidermidis and other Coagulase negative staphylococci. Mandell GL, Benett JE, Dolin R, editors. Mandell, Douglas and Bennett's principles and practice of infectious disease. 7th ed. Philadelphia: Churchill Livingstone Elsevier. p. 186.

Samant Sharvari A, Pai Chitra G, 2012. Evaluation of different detection methods of biofilm formation in clinical isolates of staphylococci. Int J Pharma Bio Sci. 3(4):724-33.

Saravanan Murugesan, Nagaraj Perumal, Surya Prakash Mahalingam, Selva Kumar Dilliappan, Padma Krishnan, 2015.Analysis of Antibiotic Resistance Genes and its Associated SCC mec Types among Nasal Carriage of Methicillin Resistant Coagulase Negative Staphylococci from Community Settings, Chennai, Southern India, JCDR, Vol. 9(8).

Sardar S, Singh M, Basireddy AS, 2015. Coagulase negative staphylococci among clinical isolates in a tertiary care 
centre. Int J pharma bio-sciences. 2015; 6(1): 229-36.

Sheikh A.F., Mehdinejad, M, 2012. Identification and determination of coagulase negative Staphylococci species and antimicrobial susceptibility pattern of isolates from clinical specimens. Afr. J. Microbiol. Res. Vol 6: 1669-74.

Winn W.C., Allen, S.D., Janda, W.M., Koneman, E.W., Procop, C.W., Schreckenberger, P.C., et al., 2006. Gram positive cocci Part I: Staphylococci and related Gram positive cocci. In: Koneman's colour atlas and textbook of diagnostic Microbiology, 6th edn. Lippincott Williams and Wilkins, USA. Pp. 62373.

Xiao Xue Ma, En Hua Wang, Yong Liu and En Jie Luo, 2011.Antibiotic susceptibility of coagulase-negative staphylococci (CONS): emergence of teicoplanin non-susceptible CONS strains with inducible resistance to vancomycin. Journal of Medical Microbiology. 60, 1661-1668

\section{How to cite this article:}

Radhika Katragadda and Sowmya A. Venkateswaran. 2018. A Study on Correlation of Antimicrobial Resistance Pattern and Biofilm Formation among Coagulase Negative Staphylococcus Isolates. Int.J.Curr.Microbiol.App.Sci. 7(11): 989-999. doi: https://doi.org/10.20546/ijcmas.2018.711.114 